\title{
Tissue Engineering
}

\section{Functional Assessment and Clinical Outcome}

\author{
STEVEN A. GOLDSTEIN \\ Orthopaedic Research Laboratories, Department of Orthopaedic Surgery, \\ University of Michigan, Ann Arbor, Michigan 48109, USA
}

\begin{abstract}
The issues that should be considered as part of the design and evaluation of tissue engineering constructs with respect to their targeted clinical application are reviewed. This paper provides a general framework for the process of bringing tissue-engineering constructs from the laboratory bench to the patient's bedside, rather than presenting a detailed review of the engineering or biologic principles or mechanisms that are necessary for successful tissue engineering. Many of the principles are animated by using examples from current studies being developed in my laboratory or those of my collaborators. In all likelihood, multiple solutions or approaches will be found that lead to successful tissue-engineering constructs. The focus here is on the identification of critical parameters to be considered rather than specific design solutions. The review is therefore organized to reflect feasible sequences of activities formulated to take tissue engineering from concept to clinical reality.
\end{abstract}

KEYWORDS: tissue engineering; bone graft substitute; gene-activated matrix

\section{CONSTRUCT CONCEPTUALIZATION}

Successful tissue engineering begins with a clear and precise definition of the clinical demand or problem being addressed. Regardless of whether the application is focused on replacing or augmenting failing heart tissue or on creating bone graft substitute materials, the design objective should begin with the delineation of the multiple specific attributes of the identified clinical problem. The incidence, prevalence, and demographics of the clinical problem need to be identified, and an appreciation for its complexity, with respect to a therapeutic intervention, needs to be developed. The current treatment options or standard of care must be accurately identified, since the new tissue-engineering solution must provide some advantage over the current therapy to merit investment in its development. It is also important to identify any unusual temporal factors associated with either the treatment or potential clinical evaluation. Most importantly, clarity in identifying the clinical problem is also the first step towards defining the required functional properties of the tissue-engineering solution. It is important to begin by identifying these targeted

Address for correspondence: Steven A. Goldstein, Ph.D., Orthopaedic Research Laboratories, Room G161, Mail Stop 0486, 400 North Ingalls, Ann Arbor, MI 48109-0486. Voice: 734-7639674; fax: 734-647-0003.

stevegld@umich.edu

Ann. N.Y. Acad. Sci. 961: 183-192 (2002). @ 2002 New York Academy of Sciences. 
functional properties in a quantitative manner, since they will become the reference of comparison for outcome measures that will be used to qualify the tissue-engineering construct as being successful or unsuccessful. For example, if the tissue-engineering target is bone replacement, the required functional properties might be categorized as follows:

Biomechanical Behavior of Bone

Material properties

Architecture/geometry/morphology

Organ level mechanical function

\section{Biologic Function}

Regulatory maintenance

Participation in physiologic homeostasis

Capacity for repair, adaptation and remodeling

As will be noted below, the critical targeted functional properties identified for specific tissue-engineering construct designs are important for guiding the design phase or discovery phase, as well as for providing specific reference variables to use as outcome measures in preclinical and eventual clinical trials.

\section{DESIGN AND FUNCTION HIERARCHY}

Following a paradigm similar to that used to solve an engineering design problem, the next phase associated with successful tissue engineering is focused on characterizing the specific design attributes and technology that will serve as the backbone of the construct under consideration. I have found it particularly valuable to consider the design attributes and targeted functions within a hierarchical framework. This concept can be demonstrated in FiguRE 1, by reviewing the images used in designing a bone tissue-engineering construct.

The targeted clinical property is a bone that has mechanical properties and geometry that enable the patient to ambulate and participate in activities of daily living. While, at the organ level, the bone must provide these macroscopic properties, bone structure and function can be decomposed into multiple hierarchical scales. These scales range from tissue morphology through the extracellular matrix to the included cells, and finally the intracellular machinery responsible for orchestrating gene expression and protein synthesis. The only way to attain the targeted organ level properties is through appropriate interactions of all of the constituents within each architectural hierarchical level as well as the correct integration of signals or properties across scales. This perspective may be valuable in that it can provide a guide for not only design conceptualization, but also evaluation of the function of constructs through a series of logical sequential tests at multiple scales.

In practice, the ability to perform this segmentation can faciliatate a design optimization process. Since all tissue engineering constructs involve the use of cells, matrices, and biofactors, this hierarchical paradigm can be used to assess how the source or manipulation of cells (in vivo or ex vivo, for example), the design of the matrices, and the choice of biofactors can influence the behavior of the construct. At the conclusion of this design phase, the hierarchical analyses, both within scale and 

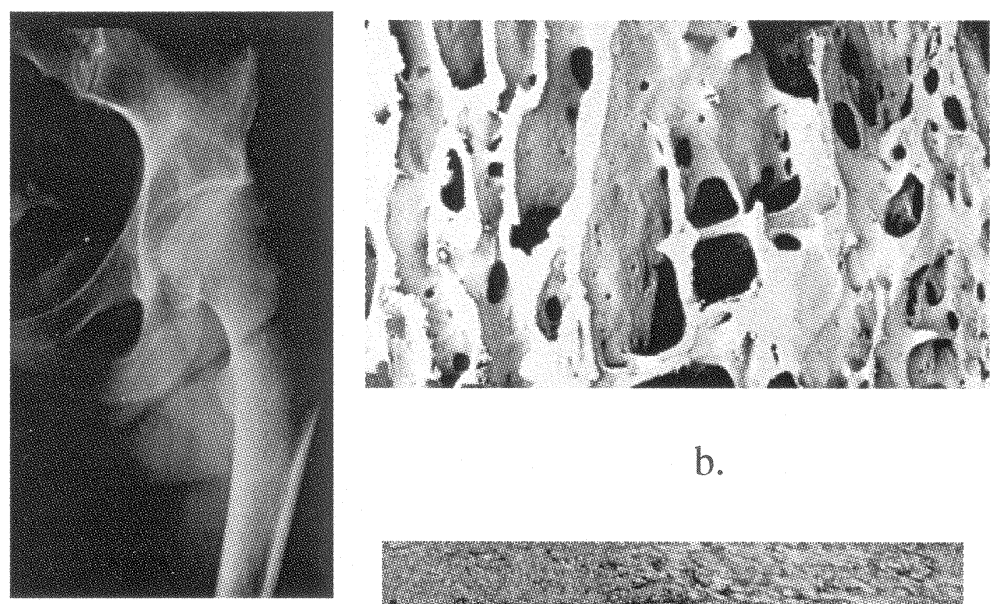

b.

a.

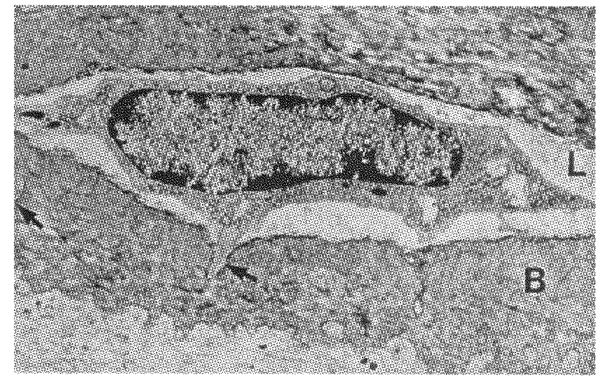

c.

FIGURE 1. Simplified hierarchical pattern of bone. Clinical function is defined at the organ level (a) as depicted by the function of the whole femur. The function of the femur, however, is dependent on the properties/function of the bone tissue architecture and properties (b), which is dependent on the cell/matrix interactions (c).

across scale, provide the proof of concept for the technology. Most often, this proof of concept involves cellular, molecular, or physiologic analyses in vitro and, importantly, in vivo.

Most investigators begin the in vivo verification of their technology in small animals with a particular focus on those biomechanical or biologic outcome variables that are most critical with respect to the targeted functional properties that were defined as part of the clinical conceptualization. This is also the stage at which a determination can be made concerning the potential of the technology to progress towards clinical utility. With convincing proof of concept and early in vivo demonstration of functional efficacy, transition to pivotal preclinical studies is often warranted.

\section{DESIGN OF PIVOTAL PRECLINICAL STUDIES}

Typically, the development of a tissue engineering construct requires the performance of several preclinical studies prior to evaluation in human subjects. As dis- 
cussed earlier, some preclinical studies are a part of the evaluation of proof of concept and are followed by expanded studies that are designed to evaluate efficacy in a relevant or well-accepted model. Usually, parallel studies are also performed to evaluate safety. These safety studies are frequently performed by third-party contract laboratories that have extensive experience with assays to evaluate toxicity and other safety-related issues.

For many tissue-engineering constructs, the pivotal preclinical studies designed to demonstrate efficacy involve a transition from the use of small animals to largeanimal models. While some of the attributes of the small-animal models will be discussed below, the need for large animals may be dependent on a need to evaluate responses of the construct under conditions that better simulate a physiologic match with the human clinical condition. The preclinical studies typically include an evaluation of dose or utilization profile, and success is significantly dependent on choosing appropriate endpoint measures. The safety studies, in contrast, are focused on evaluating toxicity, either locally or systemically, bioavailability, and the relationship between bioavailability, toxicity, and dose. Often, the safety studies can also provide assessment of the effects of storage or sterilization on the properties of the construct.

The designs, as well as the success of preclinical studies, are dependent on a number of issues. These include:

(1) the experimental model;

(2) functional assays;

(3) dose response;

(4) correspondence of preclinical and clinical variables; and

(5) safety considerations and assessments.

\section{Experimental Model}

The appropriate choice of an experimental model is critical to the success of the preclinical studies. Part of the criteria associated with choice or design of the experimental model is related to the targeted properties and, often, the expected commercial market of the tissue-engineered construct. For example, if the tissue-engineered construct is designed for a very specific clinical application, and a market strategy includes a relatively narrowly defined use, then the experimental model should be designed to simulate the clinical condition that is targeted. On the other hand, if the goal is to seek approval of a construct to be used in a broad range of clinical applications, then the experimental model may not precisely resemble any one specific clinical condition, but instead be general enough such that the results can be translated to this broad set of clinical applications. Examples of these two design objectives are illustrated as follows:

\section{Specific Clinical Application}

An example of a specific clinical application is the development of a tissue-engineered bone graft substitute for use in augmentation of spine fusions. The expected clinical use of the graft substitute might also include incorporation within a specialized device to enhance the spinal fusion. Given the specificity of this clinical target, the experimental design would likely utilize an animal model of spine fusion at a lo- 
cation that might be similar to that of human clinical need and would also use a device that would be similar to the device used in human patients.

\section{Broad Clinical Application}

In contrast, development of a bone-tissue-engineered bone graft substitute material for enhancing fractures or filling bony defects might be intended for use in any defect in long bones. This broad application requires an animal model that includes a defect that supports a healing cascade that simulates a response expected to occur in many different sites.

In addition to a careful choice of animal model based on simulating clinical conditions, it is perhaps even more critical to consider the biologic and physiologic compatibility of the chosen animal to human physiology. Using the example of the bone graft substitute, the animal model chosen should be evaluated for its ability to simulate human bone chemistry, metabolic turnover, and morphology.

\section{Quantitative Functional Assays}

Quantitative and reproducible functional assays must be developed and utilized in the pivotal preclinical study. The quantitative aspects of these functional assays cannot be underestimated, since without highly quantitative assays, the ability to statistically evaluate the efficacy of the tissue-engineering construct may be compromised. In fact, the overall statistical robustness of both the experimental model and the experimental design of the study that utilizes the model must be considered carefully. It is recommended that investigators seek consultation with biostatistical experts.

\section{Preclinical versus Clinical Studies}

As noted several times earlier in this text, the final evaluation of a tissue-engineering construct involves clinical studies that will include a number of outcome measures to assess the efficacy and potential safety of the constructs. Considering that many of these tissue-engineering constructs are based on cellular or molecular mechanisms for incorporation and regeneration, the ability to have quantitative functional assays is limited to existing imaging modalities or minimally invasive or noninvasive measures. This is in stark contrast to preclinical studies in which the animals can be sacrificed and assays ranging from macroscopic to molecular in scale can be performed in highly quantitative ways. This limitation in translating studies into the clinical arena provides an incentive for additional considerations in the design of the preclinical studies. Investigators should consider developing surrogate assays as part of the preclinical studies. In other words, the preclinical studies should include outcome measures that are similar to those that are anticipated to be utilized in the clinical studies. For example, the evaluation of incorporating a bone graft substitute in a clinical setting might include radiographs or even CT scans. In the preclinical study, however, the bone can be extracted from the animal and much higher resolution imaging technologies and even histologic technologies can be utilized. In the preclinical studies, therefore, it would be very advantageous to develop correlations between microscopic-based measures that can be taken from excised bones and noninvasive imaging techniques such as radiographs and CT scans. If robust corre- 
lations are demonstrated, the radiographs or CT scans can be utilized as surrogate functional assays. This approach and specific attention paid towards the development and assessment of these surrogate assays in the preclinical phase may substantially enhance the ability to have effective assays for the eventual clinical studies. From a functional tissue-engineering perspective, this is particularly true when the mechanical properties of the constructs are considered. There are very few, if any, assays available to noninvasively characterize mechanical properties of tissueengineering constructs. Therefore, a successful outcome depends on the development of surrogate assays for these mechanical properties during the preclinical studies.

The principles necessary to move tissue-engineering construct design from concept to the clinic have been summarized, albeit briefly. In an effort to more clearly demonstrate these principles, a more complete example will be provided below in a sequence of descriptions following the paradigm described earlier.

\section{EXAMPLE: TISSUE ENGINEERING APPROACH FOR DEVELOPMENT OF A BONE GRAFT SUBSTITUTE}

The purpose of the following section is to demonstrate the conceptual development of a bone tissue-engineered construct within the context of functional assessment during progression from the bench to the bedside.

\section{Construct Conceptualization}

There are many clinical conditions, ranging from severe trauma to reconstructive procedures, that necessitate the use of bone grafts for enhancing of healing or insuring that healing occurs. Two specific contrasting clinical needs can be defined. The first is the segmental loss of bone after substantial trauma, most often in long bones. Bone grafting has become a frequent and important part of treating these significant defects. A contrasting clinical need of interest involves procedures requiring fusion in the spine. There are a variety of clinical indications where spinal fusion becomes a therapeutic choice, but it has been demonstrated that the success rate of spinal fusion is limited and the use of bone graft to augment spine fusion is warranted. Without going into detail, investigations in the field have established a substantial need for development of bone graft substitutes. Prior work in the field has also established that the desired targeted properties of these substitutes include: (1) physical and chemical constituency that is compatible and, if possible, replicates normal bone; (2) a large surface-to-volume ratio for promoting cell recruitment and deposition; (3) the ability to fill large and potentially irregular volumes; (4) significant short-term mechanical integrity followed by an evolution of long-term properties that become equal to those of native bone; (5) a compatible environment for both osteoblast and osteoclast function such that normal bone formation and subsequent remodeling can occur; and (6) the capacity to support the eventual development of mechanical properties equal to those of normal bone, while re-establishing normal modeling and adaptational mechanisms for long-term maintenance. 


\section{Design of the Tissue-Engineering Approach}

The strategic technology that was developed involves the local delivery of DNA in a therapeutic modality to stimulate the regenerative process of bone. More specifically, a three-dimensional matrix capable of supporting associated plasmid DNA encoding for a bone-promoting protein can be physically placed into a bone defect site or into a device being utilized to stimulate bone regeneration and repair. This technology includes the development of what has been named a gene-activated matrix (GAM), which includes the delivery matrix and encoded DNA. Upon insertion into a wound site, the construct promotes recruitment and migration of repair cells that have come into contact in situ with the incorporated DNA. Upon contact with DNA, the cells may be transfected and eventually synthesize and express the bonepromoting protein. In a sense, these repair cells are converted into internal bioreactors to create the local stimulating factor that enhances the bone regeneration.

This technology and its assessment has been described elsewhere. ${ }^{1-3}$ While the details of these studies will not be presented, a brief summary of the findings will be presented.

As is described in the work cited above, cellular and molecular data supporting this concept have been presented and the proof of concept has been demonstrated. The proof of concept included not only in vitro studies, but also a series of smallanimal studies demonstrating that the transfection can occur in vivo and can even promote the formation of bone. As a result, the studies demonstrated sufficient efficacy to warrant the design and implementation of pivotal preclinical studies.

\section{Pivotal Preclinical Studies}

Several specific, pivotal preclinical studies were made. A small-animal study in rats was designed that involved the creation of a defect in the femoral diaphysis, representing a complex and difficult fracture to heal. Placement of the gene-activated matrix in this defect was monitored during the life of the animal, post surgery, by radiographs similar to those that would be utilized in a clinical study. At sacrifice, the treated bones were extracted and analyzed mechanically, histologically, and by high-resolution imaging techniques. The results demonstrated that bone was formed by this technology and that its morphology, and even mechanical integrity, were indicative of efficacy.

The small-animal study set the stage for a larger-animal study that utilized a defect in the tibia of large dogs. The rationale for moving from the small animal to the large animal involved recognition that the physiology of rat bone differs from human bone in its nutritional makeup and its ability to be remodeled. The large-animal model (canine) involved the creation of a defect in the tibia that was very difficult to heal, therefore providing a robust test for the technology. Without going into details, the bone-generating delivery system was surgically placed in the defects, radiographs were taken to follow the progression of healing, and at sacrifice the bone was evaluated mechanically, histologically, and morphologically. Again, the data suggested great promise and are beginning to support necessary efficacy that may lead to clinical trials.

Finally, another clinical model was developed, in a study designed to test the efficacy of the constructs in modeled spinal fusion. This model involved the use of 


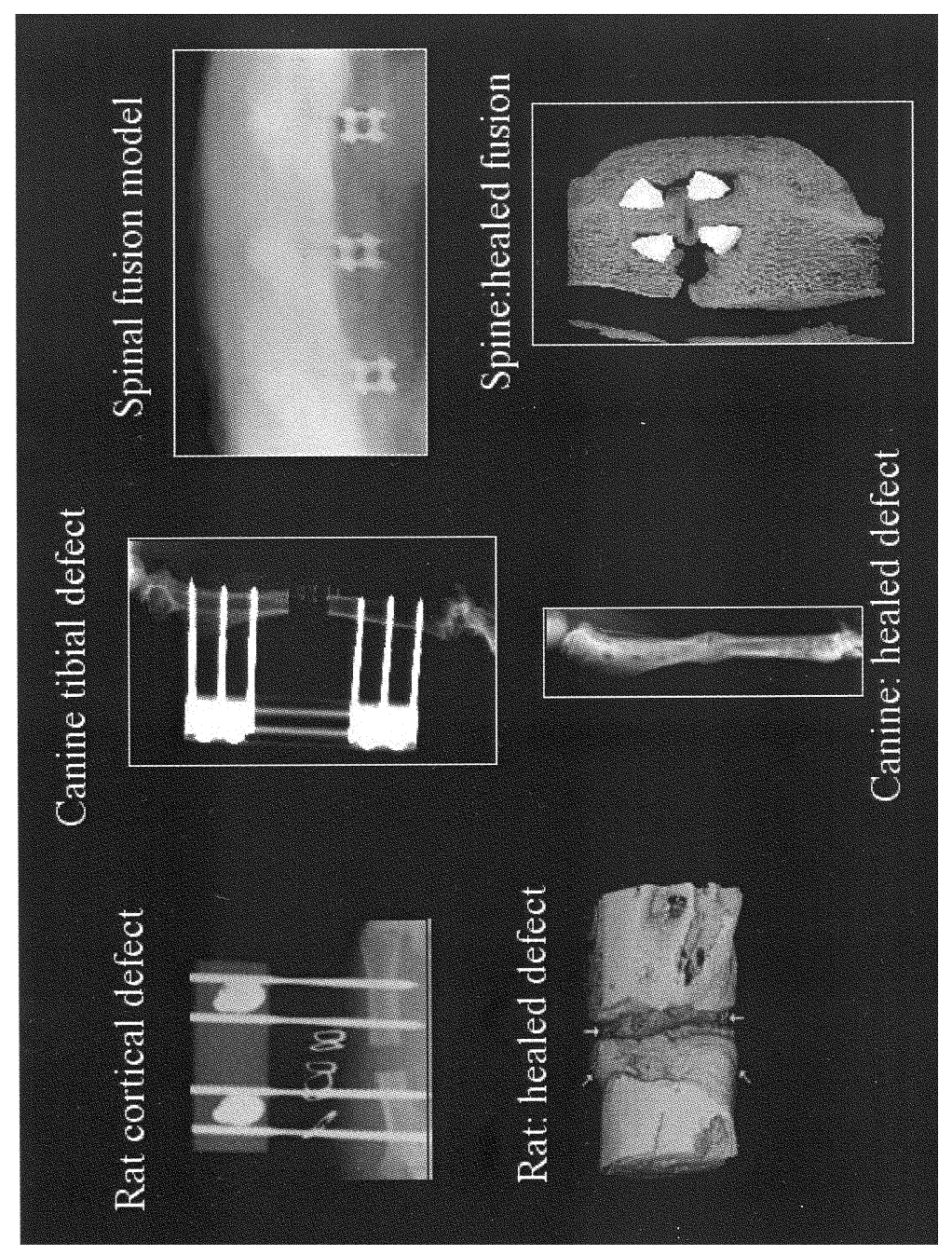

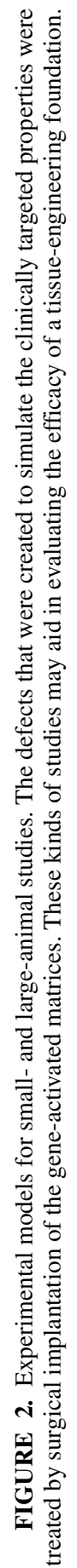


sheep, whose spines are similar to those of humans in size and morphology and, thus, a device needed to augment the spinal fusion could be used. This is a case of a very specific animal model that mimics a specific clinical need. The study actually simulated three spinal fusions in each animal. The evaluation occurred after sacrifice of the animal, when the experimental region could be removed and analyzed using histologic techniques as well as high-resolution imaging, including electron microscopy. Once again, the data were supportive of the move forward toward clinical trials as this very clinically specific model provided direct correspondence to a potential clinical use.

In the design of these models and their outcome measures, the targeted clinical utility as well as the framework of future clinical studies were considered. The functional assays ranged from biomechanical testing to sequential imaging. In illustration of this example, models and sample results are shown in FIGURE 2.

\section{SUMMARY}

This paper present some of the issues to be considered when designing experiments and methodologies to bring tissue-engineering constructs from concept to the clinic. Although they may be inferred at several points, the regulatory requirements have not been described, but regulatory issues provide a very important additional guide to experimental design and development of functional assays. In a very general sense, regulatory agencies such as the Food and Drug Administration have specific requirements for the outcome of both preclinical efficacy and safety studies, as well as requirements for establishing manufacturing composition, methods, and controls. The selection and design of the outcome variables for the clinical studies, as well as an assessment of risk benefit will also be of critical importance to the regulatory review process.

The principles that must be considered for successful tissue engineering include the following:

(1) comprehensive description and characterization of the properties of the native tissue;

(2) thorough design optimization based on clinical need and targeted functional properties;

(3) conceptualization of the tissue-engineering construct within a hierarchical paradigm that includes functional assessment across multiple scales from the cellular and molecular level, through the organ level;

(4) assessment of both biomechanical and biologic function and adaptation; and

(5) the development of preclinical studies that include considerations of characterizing surrogate variables in the preclinical studies that may aid in functional assessment during the clinical phase.

\section{ACKNOWLEDGMENTS}

The bone-tissue engineering example using the gene-activated matrix is based on research supported by Selective Genetics, Inc. [The author has an affiliation with Se- 
lective Genetics and discloses that the results on continued studies using the geneactivated matrices may result in the potential for financial gain.]

\section{REFERENCES}

1. Bonadio, J., E. Smiley, P. Patil \& S.A. Goldstein. 1999. Localized, direct plasmidgene delivery in vivo: prolonged therapy results in reproducible tissue regeneration. Nat. Med. 5: 753-759.

2. FANG, J., Y.-Y. Zhu, E. Smiley, et al. 1996. Stimulation of new bone formation by direct transfer of osteogenic plasmid genes. Proc. Natl. Acad. Sci. USA 93: 5753-5758.

3. GoldSTEIN, S.A. 2000. In vivo nonviral delivery factors to enhance bone repair. CORR 379S: S113-S119. 